Article

\title{
Dynamic Cerebral Autoregulation and Critical Closing Pressure in Experimental Human Endotoxemia and Sepsis Patients
}

\author{
Judith Maria Dimphena van den Brule ${ }^{1, *}$, Christiaan Ruud van Kaam ${ }^{1}$, \\ Guus Pieter Leijte ${ }^{1,2}$, Roeland Stolk ${ }^{1,2}$, Peter Pickkers ${ }^{1,2}$, \\ Johannes Gerardus van der Hoeven ${ }^{1,2}$, Matthijs Kox ${ }^{1,2}$, \\ Cornelia Wilhelmina Elisabeth Hoedemaekers ${ }^{1}$ \\ 1 Department of Intensive Care Medicine, Radboud University Medical Center, \\ 6500 HB Nijmegen, The Netherlands \\ 2 Radboud Center for Infectious Diseases (RCI), Radboud University Medical \\ Center, 6500HB Nijmegen, The Netherlands \\ * Correspondence: Judith Maria Dimphena van den Brule, \\ Email: Judith.vandenBrule@radboudumc.nl; Tel.: +31-24-3617273; \\ Fax: +31-24-3541612.
}

\begin{abstract}
Background: To investigate the influence of systemic inflammation on dynamic cerebral autoregulation and vascular tone during experimental human endotoxemia and sepsis.

Methods: Healthy volunteers received $3 \mathrm{~h}$ continuous infusion of LPS (c-LPS, $4 \mathrm{ng} / \mathrm{kg}, n=11$, Clinicaltrials.gov NCT02922673) or a bolus of LPS (b-LPS, 2 ng/kg, $n=8$, Clinicaltrials.gov NCT02675868) and 10 sepsis patients were studied. Mean arterial pressure (MAP) and cerebral blood flow velocity (CBFV) were monitored simultaneously. Cerebral autoregulation was analysed by transfer function analysis (TFA). Critical closing pressure ( $\mathrm{CrCP}$ ) was estimated as a measure of cerebral vascular tone.
\end{abstract}

Results: c-LPS resulted in a more pronounced and prolonged plasma cytokine response compared with b-LPS. MAP decreased from $89 \pm 3$ to $75 \pm 2 \mathrm{mmHg}(p<0.05)$ and from $91 \pm 2$ to $77 \pm 3 \mathrm{mmHg}(p<0.001)$ in the

\section{G Open Access}

Received: 16 April 2019 Accepted: 22 May 2019

Published: 28 May 2019

Copyright $(02019$ by the author(s). Licensee Hapres,

London, United Kingdom. This is an open access article distributed under the terms and conditions of Creative Commons Attribution 4.0 International License. c-LPS and b-LPS groups, respectively. MAP in sepsis patients was $65 \pm 4$ mmHg. TFA in both LPS groups showed no significant changes over time in coherence, gain and phase. Phase in sepsis patients was lower compared with both LPS groups (7 (2 to 33) [median (interquartile range)] in sepsis versus 57 (36 to 74) in the c-LPS ( $p=0.02$ ) and 53 (43 to 64) degrees in the b-LPS group $(p=0.01)$ ). CrCP decreased from $49 \pm 2$ to $41 \pm 2 \mathrm{mmHg}$ $(p=0.16)$ in the c-LPS and from $50 \pm 2$ to $42 \pm 2 \mathrm{mmHg}$ in the b-LPS group ( $p<0.01$ ), and was $36 \pm 2 \mathrm{mmHg}$ in sepsis patients.

Conclusions: Dynamic cerebral autoregulation is impaired in sepsis patients, but remains intact during experimental human endotoxemia. $\mathrm{CrCP}$ decreased during endotoxemia and was low in sepsis, reflecting decreased vascular tone. This indicates activation of a cerebrovascular 
adaptive process, also during experimental endotoxemia with intact cerebral autoregulation.

Trial Registration: Clinicaltrials.gov NCT02922673 and Clinicaltrials.gov NCT02675868.

KEYWORDS: sepsis; human endotoxemia model; dynamic cerebral autoregulation; critical closing pressure

\section{INTRODUCTION}

Sepsis is defined as life-threatening organ dysfunction caused by a dysregulated host response to infection [1]. The brain is frequently affected in sepsis and sepsis-associated encephalopathy (SAE) is associated with increased mortality, prolonged hospital stay and long-term cognitive decline [2]. The pathophysiology of SAE is multifactorial, and includes diffuse neuroinflammation, abnormalities in cerebral perfusion, and excitotoxicity [2].

Dynamic cerebral autoregulation is a mechanism that maintains a stable cerebral blood flow (CBF) by dampening the effects of alterations in arterial blood pressure (ABP) through cerebral arteriolar vasodilatation and vasoconstriction. Under healthy circumstances, spontaneous fluctuations in $\mathrm{ABP}$ are damped to preserve a constant $\mathrm{CBF}$, in order to maintain cerebrovascular homeostasis. However, this so-called dynamic cerebral autoregulation is frequently impaired in sepsis, especially in the first few days [3-5]. A disturbed cerebral autoregulation may represent an important mechanism in the pathophysiology of SAE, rendering the brain more vulnerable to changes in perfusion pressure [4]. The most frequently used method to quantify dynamic cerebral autoregulation is transfer function analysis (TFA), with the calculation of coherence, gain and phase [6]. TFA uses beat-to-beat blood pressure variation as input signal [6]. The coherence function tests the linearity of the relation between input and output. The gain quantifies the damping effect between the input and output of the transfer function. The phase shift of a waveform represents the displacement of this waveform relative to another waveform with the same period. Gain and phase are the parameters from which dynamic cerebral autoregulation can be determined [6].

In addition to dynamic autoregulation, the critical closing pressure ( $\mathrm{CrCP}$ ) is another frequently used tool in cerebral vascular research. $\mathrm{CrCP}$ is defined as the lower limit of ABP below which vessels collapse and flow ceases [7]. CrCP allows estimation of changes in cerebrovascular tone and minimal cerebral perfusion pressure to prevent collapse of vessels and ischemia [7].

The experimental human endotoxemia model is a highly standardized and controlled model of systemic inflammation induced by intravenous administration of lipopolysaccharide (LPS) in healthy volunteers that captures many hallmarks of sepsis [8]. Previous work has demonstrated 
that administration of $2 \mathrm{ng} / \mathrm{kg}$ LPS results in an improved dynamic autoregulation [3,9-11]. These effects differ from those observed in sepsis patients, in whom dynamic autoregulation is impaired in the first few days and restores later on [3,4,12-18]. The observed differences between the human endotoxemia model and sepsis may be related to the use of a relatively low LPS dose or the fact that LPS is administrated as a single bolus or during $4 \mathrm{~h}$, whereas sepsis patients are exposed to micro-organisms and their toxins for a more sustained period. We previously showed that endotoxemia induced by continuous infusion of LPS at a total dose of $4 \mathrm{ng} / \mathrm{kg}$ results in a significantly more pronounced and prolonged inflammatory response compared to bolus administration of $2 \mathrm{ng} / \mathrm{kg}$ LPS, which may better recapitulate the response observed in septic patients [19]. Therefore, continuous infusion of LPS may also result in a cerebrovascular profile that better resembles the situation in sepsis. This would enable the use of such a "continuous human endotoxemia model" for investigation of new therapies aimed at maintaining cerebrovascular homeostasis in sepsis.

In the present study, we investigated dynamic cerebral autoregulation and CrCP in healthy volunteers following continuous or bolus administration of LPS, and compared these responses to those observed in sepsis patients. Some clinical data (ABP, heart rate, temperature), cerebral blood flow velocity (CBFV) and $\mathrm{CrCP}$ of the 8 endotoxemia subjects who received a bolus administration of LPS and of the 10 sepsis patients were previously reported [20]. So in this study we also investigated dynamic cerebral autoregulation and $\mathrm{CrCP}$ following continuous (and higher total dose) administration of LPS to observe if this dose regime better resembles the situation in sepsis.

\section{METHODS}

\section{Study Design, Setting, Subjects and Patients}

We performed a prospective observational study in healthy male volunteers during experimental endotoxemia induced by 2 different dosing regimens, and an observational study in sepsis patients. Data were obtained from a total of 19 healthy, non-smoking, male volunteers, aged 18 to 35 years, 11 of whom were included in a human endotoxemia study employing continuous infusion of LPS, and 8 of whom were included in a human endotoxemia study employing single bolus administration of LPS. All subjects provided written informed consent and experiments were in accordance with the Declaration of Helsinki and Good Clinical Practice guidelines, and approved by the local ethics committee Commissie Mensgebonden Onderwoek region Arnhem-Nijmegen with document numbers NL57410.091.16 and NL53411.091.15 on 29 August 2016 and 6 January 2016, respectively. Project identification codes are: Clinicaltrials.gov NCT02922673 for continuous infusion of LPS; Clinicaltrials.gov NCT02675868 for single bolus administration of LPS. 
Subjects were screened prior to inclusion, and had a normal physical examination, electrocardiography and routine laboratory values. Also, 10 patients with septic shock, older than 18 years, were included. The local Institutional Review Board waived the need for informed consent for these sepsis patients. Septic shock was defined by the international sepsis definition conference [21]. Sepsis patients were treated according to international management guidelines [22]. All sepsis patients received sedating medication and were mechanically ventilated to achieve normocapnia. Exclusion criteria were an irregular heart rhythm or an insufficient transtemporal bone window.

\section{Experimental Human Endotoxemia}

Purified LPS (continuous infusion study: Escherichia coli 0:113, Lot no. 94332B4, List Biological Laboratories, Campbell, USA; bolus administration study: US Standard Reference Endotoxin Escherichia coli 0:113, Pharmaceutical Development Section of the National Institutes of Health, Bethesda, USA) was supplied as a lyophilized powder and dissolved in normal saline $0.9 \%$ as described previously [23,24]. For the continuous infusion study (c-LPS), a total of $4 \mathrm{ng} / \mathrm{kg}$ LPS was administered as a intravenous loading bolus of $1 \mathrm{ng} / \mathrm{kg}$ body weight at $\mathrm{T}=0$, followed by an intravenous infusion of $1 \mathrm{ng} / \mathrm{kg} / \mathrm{h}$ for a period of $3 \mathrm{~h}$ [24]. For the bolus administration study (b-LPS), LPS was administered, as described previously, as an intravenous bolus injection at a dose of $2 \mathrm{ng} / \mathrm{kg}$ body weight in 1 min at $\mathrm{T}=0$ [23].

All subjects received $1.5 \mathrm{~L}$ of $2.5 \%$ glucose $/ 0.45 \%$ saline solution in the hour before initiation of LPS administration, followed by $150 \mathrm{~mL} / \mathrm{h}$ during the first $6 \mathrm{~h}$ after LPS administration and $75 \mathrm{~mL} / \mathrm{h}$ until the end of the experiment, according to our protocol [24].

\section{Data Collection}

$\mathrm{ABP}$, heart rate, temperature and CBFV in the middle cerebral artery (MCA) were monitored during the experiment and simultaneously recorded and stored, as described in a previous manuscript [23]. All recordings were made in supine position with the head elevated $30^{\circ}$. Recordings were performed at $60 \mathrm{~min}$ before (baseline, $\mathrm{T}=\mathrm{BL}$ ), and at 150, 300 and 420 min after initiation of LPS administration in the c-LPS group and at 90 min before (baseline, $\mathrm{T}=\mathrm{BL}$ ), and at 90, 210 and $270 \mathrm{~min}$ after LPS administration in the b-LPS group. Data from sepsis patients were obtained once, under stable hemodynamic (normotensive) and respiratory (normocapnic) conditions.

Plasma levels of IL-6, IL-8, IL-10 and TNFa were determined at baseline and at $60,90,120,150,180,210,240,360$ and $360 \mathrm{~min}$ after initiation of LPS administration in the c-LPS group and at $60 \mathrm{~min}$ before LPS administration, baseline and at 60, 90, 120, 180, 240, 360 and 480 min after LPS administration in the b-LPS group. For determination of plasma levels of IL-6, IL-8, IL-10 and TNFa, ethylenediaminetetraacetic (EDTA)- 
anticoagulated blood was centrifuged immediately after withdrawal (2000× $\mathrm{g}, 10 \mathrm{~min}, 4{ }^{\circ} \mathrm{C}$ ) after which plasma was stored at $-80{ }^{\circ} \mathrm{C}$ until analysis by a multiplex Luminex assay (Milliplex, Billerica, USA).

Some clinical data (ABP, heart rate, temperature), $\mathrm{CBFV}$ and $\mathrm{CrCP}$ of the 8 endotoxemia subjects who received a bolus administration of LPS and of the 10 sepsis patients were previously reported [20].

\section{Data Analysis}

Analysis of $\mathrm{ABP}$ and $\mathrm{CBFV}$ was performed using a custom-written MATLAB script (Matlab R2014b, The MathWorks Inc., Natick, MA, USA). Mean ABP (MAP) and mean flow velocity (MFV) were obtained filtering $\mathrm{ABP}$ and CBFV with a 3th order low-pass Butterworth filter. Raw and mean data underwent visual inspection and artifact free segments of 5 min were selected for subsequent analysis.

\section{Cerebral Autoregulation}

Dynamic autoregulation was determined by transfer function analysis (TFA) and performed according to the recommendations of the international Cerebral Autoregulation Research Network (CARNet) to assess cerebral autoregulation in the frequency domain using artifact free segments of $5 \mathrm{~min}$ [6]. The TFA coherence, gain and phase were calculated for the very low (VLF, 0.02-0.07 Hz) and low (LF, 0.07-0.2 Hz) frequency bands. In addition, the average spectral power of MAP and MFV were calculated in the VLF and LF frequency bands in order to see whether the variation can be designated to a certain frequency band and whether this origin of variation changes over time in the patients [6].

\section{Critical Closing Pressure}

CrCP was estimated according to the method by Varsos et al. [25,26]:

$$
C r C P=A B P-\frac{C P P}{\sqrt{\left(C V R \cdot C_{a} \cdot H R \cdot 2 \pi\right)^{2}+1}}
$$

with CVR means cerebrovascular resistance, $C_{a}$ means compliance of the cerebral vascular bed and HR means heart rate. Cerebral perfusion pressure (CPP) is defined as ABP - ICP (intracranial pressure), however in this study ICP was not measured. Therefore mean ABP was used as an approximation of CPP, as previously described [26]. CVR was calculated by dividing ABPmean by mean flow velocity (MFV). To determine $C_{a}$, cerebral arterial blood volume (CABV) was calculated by integrating the MFV signal over time. Then $C_{a}$ was calculated by dividing the amplitude of the first harmonic of CABV by the amplitude of the first harmonic of ABP. HR was defined as the first harmonic frequency of ABP. 


\section{Calculations and Statistical Analysis}

Statistical analysis was performed using GraphPad Prism version 5.0 (GraphPad Software, La Jolla, CA, USA). Data are presented as mean \pm SD, mean \pm SEM, or median (interquartile range), according to their distribution (determined by Kolmogorov-Smirnov tests). According to the distribution of the data, within-group changes over time were analyzed with repeated measures ANOVA or Friedman tests followed by Dunnet's or Dunn's post-hoc tests. Mann Whitney U-tests were used for between-group comparisons. A $p$-value of $<0.05$ was considered statistically significant.

\section{RESULTS}

\section{Baseline Characteristics and LPS-Induced Inflammatory Response Parameters}

Baseline characteristics of the healthy volunteers participating in the experimental human endotoxemia studies and sepsis patients are presented in Table 1. SAPS 2 score in sepsis patients was 55 (49 to 77), and the APACHE 2 score was 25 (18 to 29). All patients in the sepsis population received norepinephrine (median dose 0.3 ( 0.2 to 0.5$) \mu \mathrm{g} / \mathrm{kg} / \mathrm{min}$ ).

IL-6, IL-8, and IL-10, but not TNF-a, have a higher peak level in the c-LPS group than in the b-LPS group (Figure 1).

Table 1. Demographic and clinical data at baseline of endotoxemia subjects and sepsis patients. Data are expressed as mean $\pm \mathrm{SD}$.

\begin{tabular}{lccc}
\hline & $\begin{array}{c}\text { Healthy Volunteers } \\
\text { c-LPS }(\boldsymbol{n}=\mathbf{1 1})\end{array}$ & $\begin{array}{c}\text { Healthy Volunteers } \\
\text { b-LPS }(\boldsymbol{n}=\mathbf{8})\end{array}$ & $\begin{array}{c}\text { Sepsis Patients } \\
(\boldsymbol{n}=\mathbf{1 0})\end{array}$ \\
\hline Age $(\mathrm{y})$ & $22.4 \pm 1.6$ & $23.1 \pm 3.6$ & $63.1 \pm 12.8$ \\
BMI $\left(\mathrm{kg} / \mathrm{m}^{2}\right)$ & $24.4 \pm 2.7$ & $20.5 \pm 1.8$ & $26.2 \pm 3.3$ \\
HR $\left(\mathrm{min}^{-1}\right)$ & $68.5 \pm 8.7$ & $62.3 \pm 11.3$ & $105.2 \pm 13.3$ \\
MAP $(\mathrm{mmHg})$ & $96.8 \pm 5.1$ & $90.8 \pm 5.2$ & $68.1 \pm 10.2$ \\
Temperature $\left({ }^{\circ} \mathrm{C}\right)$ & $36.4 \pm 0.6$ & $36.9 \pm 0.5$ & $37.4 \pm 1.3$ \\
\hline
\end{tabular}

Abbreviations: BMI, body mass index; HR, heart rate; MAP, mean arterial pressure.

\section{Hemodynamic Data}

MAP in the c-LPS group was $89 \pm 3 \mathrm{mmHg}$ before initiation of LPS infusion (baseline, $\mathrm{T}=\mathrm{BL}$ ) and decreased to $75 \pm 2 \mathrm{mmHg}$ at $\mathrm{T}=420 \mathrm{~min}$ $(p<0.05$, Figure 2A). MAP in the b-LPS group was $91 \pm 2 \mathrm{mmHg}$ before LPS administration $(\mathrm{T}=\mathrm{BL})$ and decreased to $77 \pm 3 \mathrm{mmHg}$ at $\mathrm{T}=210 \mathrm{~min}$ $(p<0.001$, Figure 2A). MAP in sepsis patients was lower than the lowest MAPs observed in both LPS groups ( $65 \pm 4 \mathrm{mmHg}, p<0.05$, Figure $2 \mathrm{~A}$ ). CBFV before LPS infusion was $60 \pm 4 \mathrm{~cm} / \mathrm{s}$ in the c-LPS group and $72 \pm 4 \mathrm{~cm} / \mathrm{s}$ in the b-LPS group (Figure 2B). In both LPS groups, CBFV did not change significantly following LPS administration (Figure 2B). Furthermore, CBFV in sepsis patients was comparable to that observed in healthy volunteers (57 $\pm 6 \mathrm{~cm} / \mathrm{s}$, Figure 2B). 

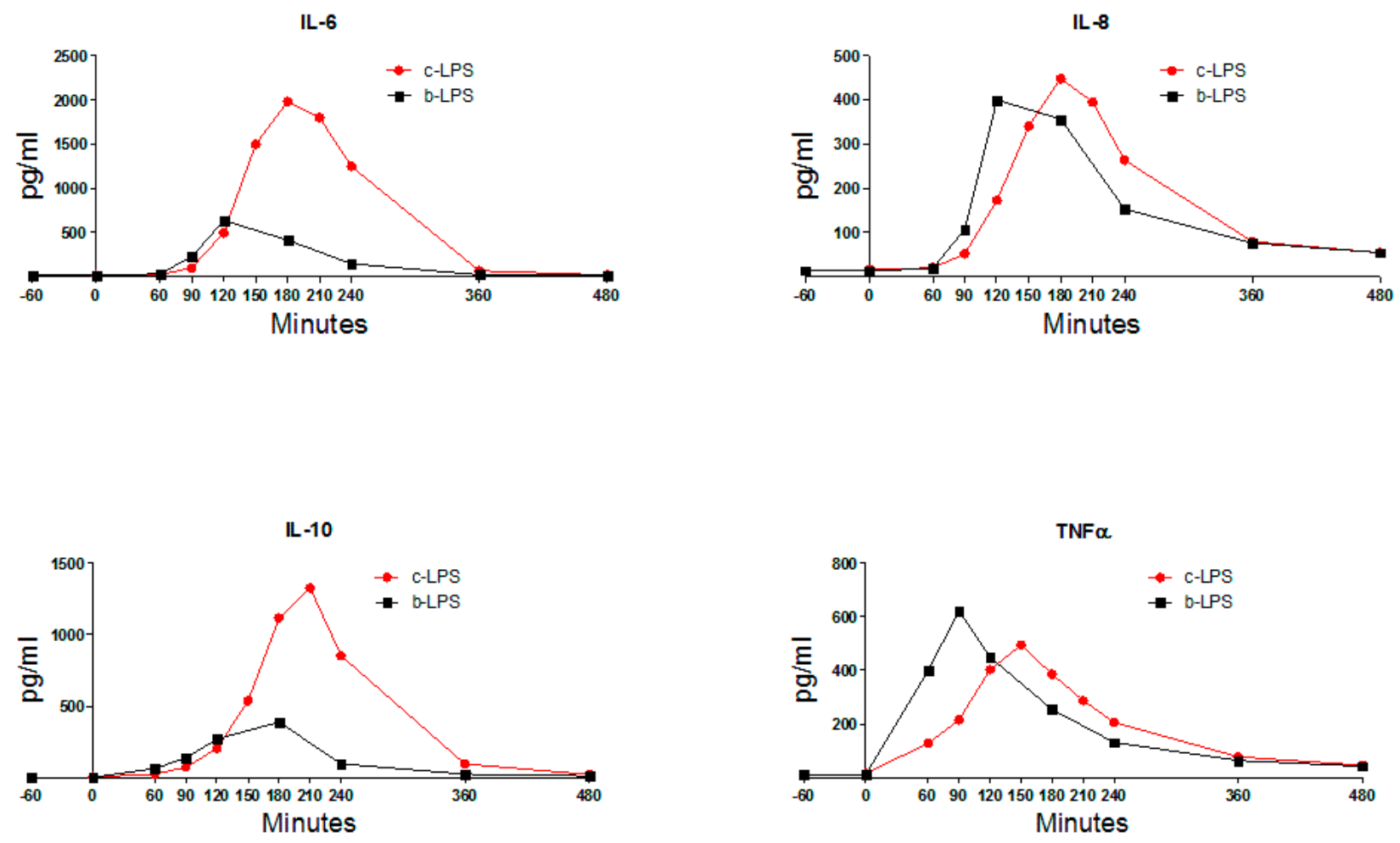

Figure 1. Plasma levels of IL-6, IL-8, IL-10 and TNFa of endotoxemia subjects. For reasons of clarity, only mean values are depicted to illustrate the differences in kinetics and magnitude of the cytokine response between continuous and bolus LPS infusion/administration. LPS infusion/administration was initiated/performed at $\mathrm{T}=0$. Abbreviations: IL-6, interleukin 6; IL-8, interleukin 8; IL-10, interleukin 10; TNFa, tumor necrosis factor a; c-LPS, continuous LPS infusion; b-LPS, bolus LPS administration.

A

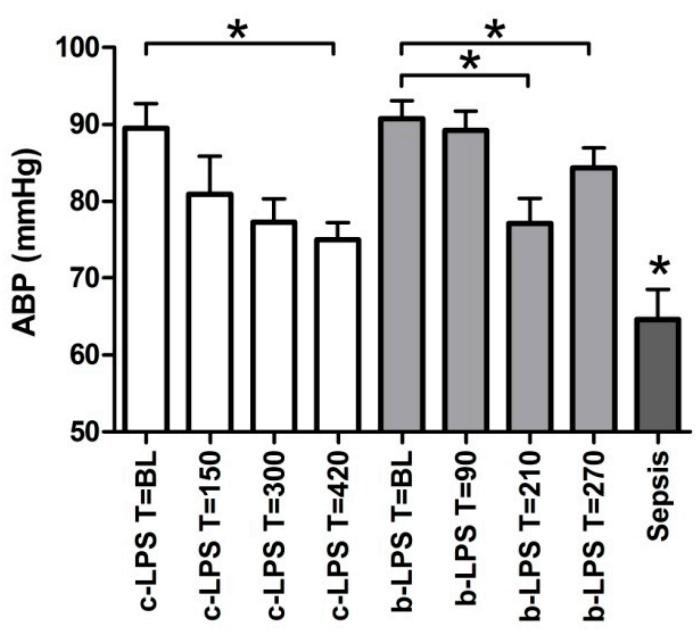

B

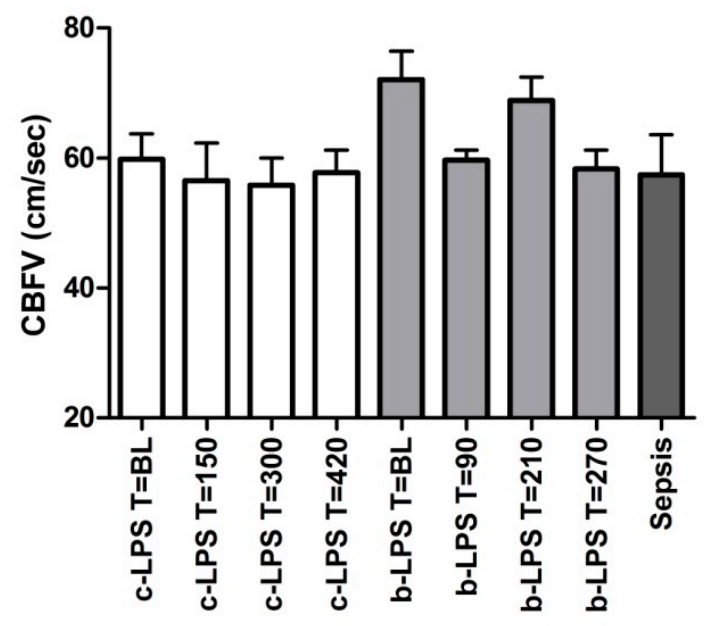

Figure 2. Arterial blood pressure (A) and cerebral blood flow (B) of endotoxemia subjects and sepsis patients. Data are expressed as mean \pm SEM (arterial blood pressure). * indicates $p<0.05 .{ }^{*}$ depicted above the sepsis patients data indicates $p<0.05$ compared to c-LPS and b-LPS at all timepoints. White bars reflect c-LPS, light grey bars b-LPS and dark grey bars sepsis patients. Abbreviations: ABP, arterial blood pressure; BL, baseline; CBFV, cerebral blood flow velocity; c-LPS, continuous LPS infusion; b-LPS, bolus LPS administration. 
In the frequency domain, neither continuous infusion nor bolus administration of LPS resulted in changes in coherence, gain and phase in the VLF and LF bands over time (Figure 3A-C, LF band data not shown). VLF coherence in sepsis patients was comparable to the coherence observed in healthy volunteers $(0.5 \pm 0.1$ in sepsis patients compared to $0.4 \pm 0.04$ in the c-LPS group at baseline and $0.4 \pm 0.1$ in the b-LPS group at baseline, Figure 3A). VLF gain in sepsis patients was higher compared to VLF gain at baseline and T $=150$ in the c-LPS group $\left(1.0 \pm 0.1 \mathrm{~cm} \cdot \mathrm{s}^{-1} \cdot \mathrm{mmHg}^{-1}\right.$ compared to $0.7 \pm 0.1(p=0.04)$ and $0.7 \pm 0.1(p=0.04)$, respectively, Figure 3B), but not significantly different from VLF gain measured at any of the time points in the b-LPS group (Figure 3B). VLF phase in sepsis patients was significantly lower compared to both LPS groups at all timepoints (7 (2 to 33) degrees, $p<0.05$, Figure 3C).

A

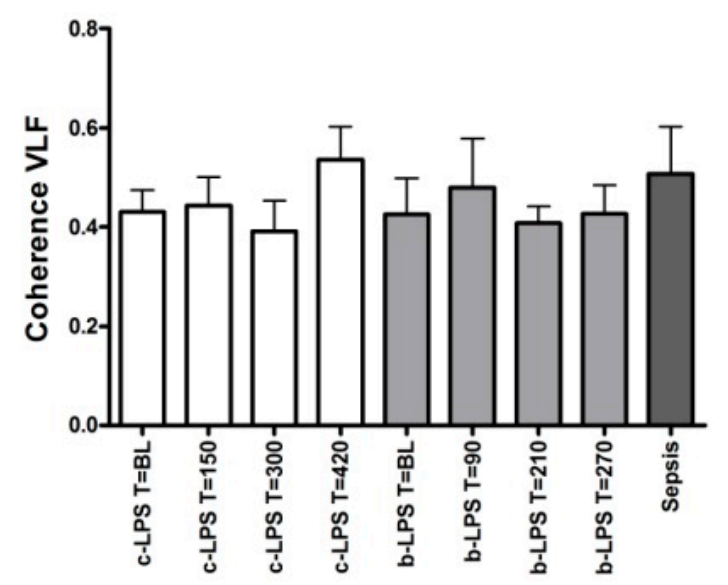

C

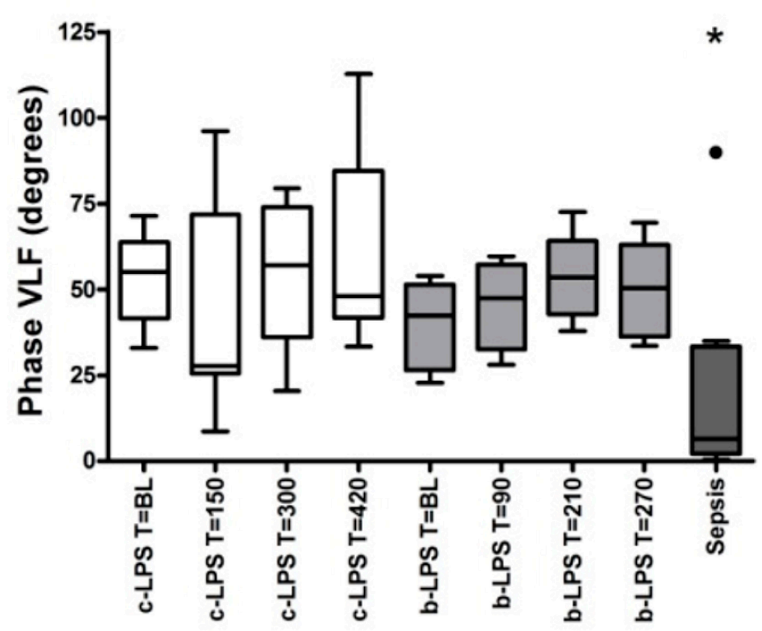

B

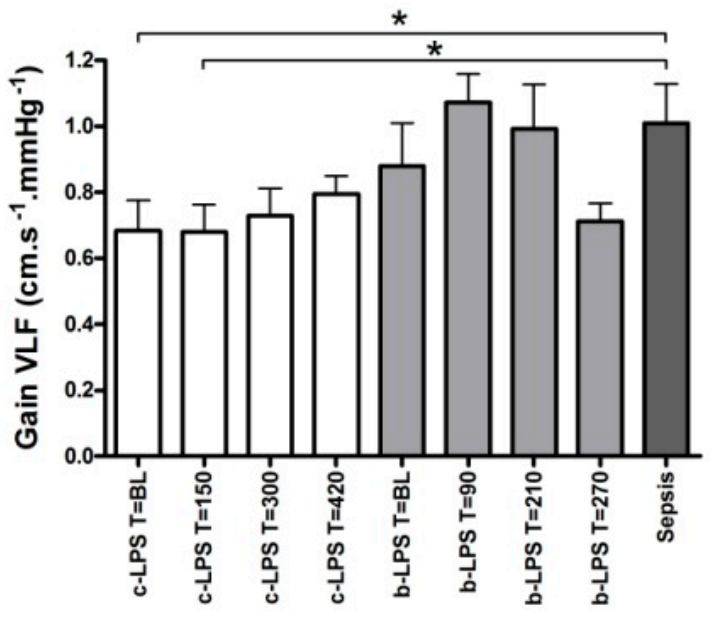

Figure 3. Coherence (A), gain (B) and phase (C) in the VLF band of endoxemia subjects and sepsis patients. Data are expressed as mean \pm SEM. ${ }^{*}$ indicates $p<0.05 .{ }^{*}$ depicted above the sepsis patients data in panel C indicates $p<0.05$ compared to c-LPS and b-LPS at all timepoints. White bars reflect c-LPS, light grey bars b-LPS and dark grey bars sepsis patients. Abbreviations: BL, baseline; VLF, very low frequency band; c-LPS, continuous LPS infusion; b-LPS, bolus LPS administration. 
No statistical differences were observed in VLF spectral power of MAP and CBFV of the c-LPS or b-LPS group over time (Figure 4A,B).

CrCP decreased in the c-LPS group from $49 \pm 2$ at baseline to $41 \pm 2$ mmHg at $\mathrm{T}=420(p=0.16)$ and in the b-LPS group from $50 \pm 2$ at baseline to $42 \pm 2 \mathrm{mmHg}$ at $\mathrm{T}=210(p<0.01$, Figure $5 \mathrm{~A})$. Sepsis patients displayed a lower CrCP compared to both LPS groups at all timepoints (36 $\pm 2 \mathrm{mmHg}$, $p<0.05$, Figure 5A).

A

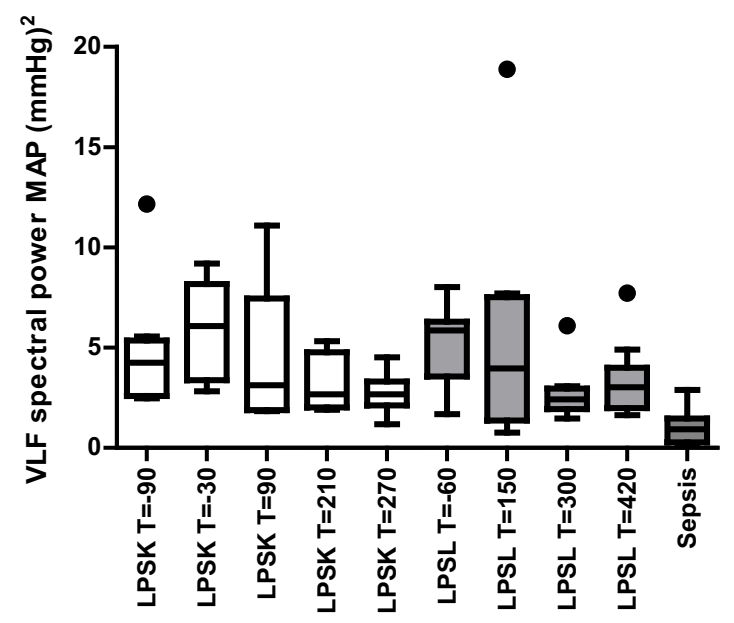

B

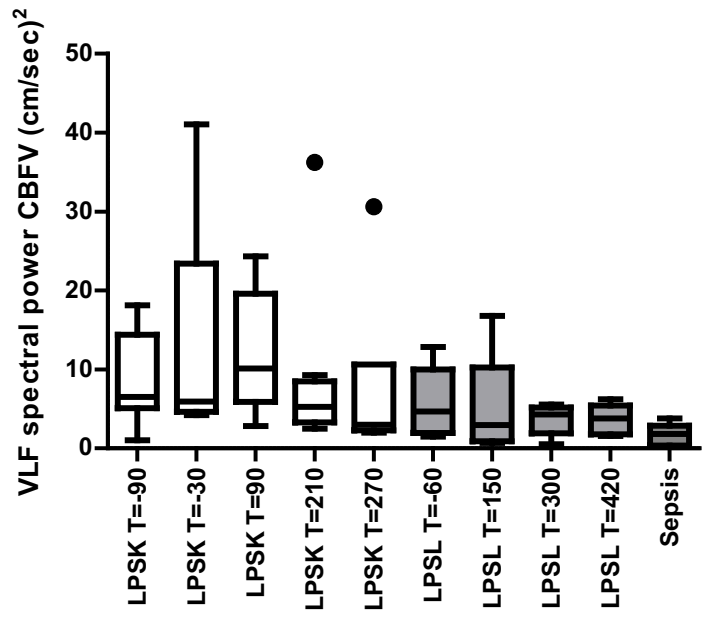

Figure 4. Spectral power in the VLF spectrum of MAP (A) and CBFV (B) of endotoxemia subjects and sepsis patients. Data are expressed as mean \pm SEM. White bars reflect c-LPS, light grey bars b-LPS and dark grey bars sepsis patients.

A

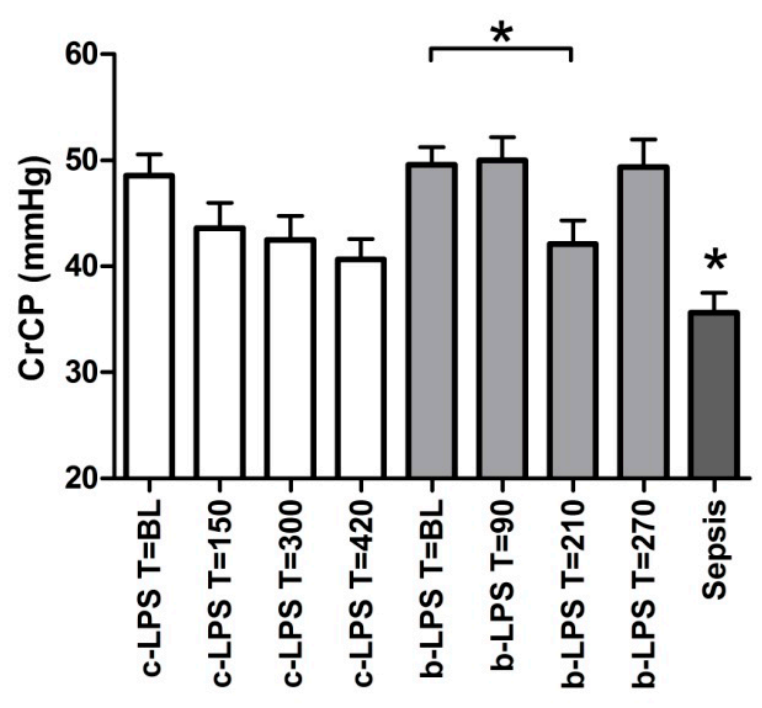

B

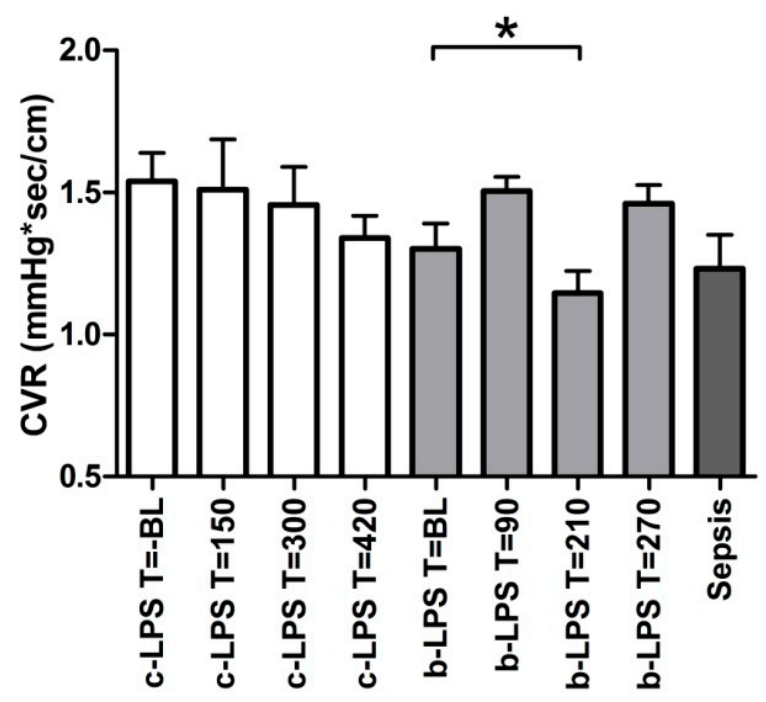

Figure 5. Critical closing pressure (A) and cerebrovascular resistance (B) of endoxemia subjects and sepsis patients. Data are expressed as mean \pm SEM. ${ }^{*}$ indicates $p<0.05 .{ }^{*}$ depicted above the sepsis patients data indicates $p<0.05$ compared to c-LPS and b-LPS at all timepoints. White bars reflect c-LPS, light grey bars b-LPS and dark grey bars sepsis patients. Abbreviations: BL, baseline; CrCP, critical closing pressure; CVR, cerebrovascular resistance; c-LPS, continuous LPS infusion; b-LPS, bolus LPS administration. 
No differences in CVR were observed in the c-LPS group over time (Figure 5B). In the b-LPS group, CVR decreased from $1.3 \pm 0.1 \mathrm{mmHg} \cdot \mathrm{sec} / \mathrm{cm}$ at baseline to $1.1 \pm 0.1 \mathrm{mmHg} \cdot \mathrm{sec} / \mathrm{cm}$ at $\mathrm{T}=210(p<0.05$, Figure 5B). CVR in sepsis was $1.2 \pm 0.1 \mathrm{mmHg} \cdot \mathrm{sec} / \mathrm{cm}$ (Figure $5 \mathrm{~B}$ ), comparable to that observed in healthy volunteers after bolus administration of LPS.

There were no correlations between plasma cytokine levels and phase in the VLF band or CrCP (data not shown).

\section{DISCUSSION}

Herein, we demonstrate that, although lower blood pressure is observed both during human endotoxemia and sepsis, dynamic cerebral autoregulation remains unchanged during endotoxemia, but is impaired in sepsis. Furthermore, CrCP decreases upon administration of LPS and is low in sepsis, indicating changes in cerebrovascular tone with a decreased lower limit of ABP below which vessels collapse and flow ceases.

Dynamic cerebral autoregulation is disturbed in septic patients and associated with the development of SAE and mortality [3,4]. In our sepsis patients this was exemplified by a lower VLF phase than found in any of our LPS groups. In our experiments however, continuous LPS infusion or bolus LPS administration did not change dynamic cerebral autoregulation. CrCP did decrease during experimental endotoxemia, indicating a decreased vascular tone. Taken together, these results indicate that despite a lower blood pressure and decreased vascular tone as signs of a vasodilatory status, cerebral autoregulation remains intact in the human endotoxemia model. In constrast, in septic shock patients, cerebral autoregulation is impaired, likely due to an exhausted compensatory cardiovascular mechanism.

Human endotoxemia is frequently utilized as a model to study the cerebrovascular effects of sepsis. Previously, administration of $2 \mathrm{ng} / \mathrm{kg}$ of LPS was shown to result in an increased phase difference as determined by TFA [3,9,10]. Two studies infused $2 \mathrm{ng} / \mathrm{kg}$ LPS over a $4 \mathrm{~h}$ period [3,9]. The third study administered the same dose as a bolus [10]. The increased phase implies a more swift response of the cerebrovascular bed to perfusion changes. This enhanced dynamic cerebral autoregulation is considered to reflect the hemodynamic changes in the earlier phases of sepsis [3,27,28]. We propose that administration of $2 \mathrm{ng} / \mathrm{kg}$ LPS (as bolus or continuous infusion) is an inadequate stimulus to induce the sepsis-related cerebrovascular changes, because dynamic cerebral autoregulation does not deteriorate as in sepsis patients.

Continuous infusion of $4 \mathrm{ng} / \mathrm{kg}$ LPS over three hours resulted in a significant decrease in MAP and a more pronounced inflammatory response compared to $2 \mathrm{ng} / \mathrm{kg}$ LPS bolus administration, as also previously described [19]. Still, dynamic cerebral autoregulation remained unaffected. This stable cerebral autoregulatory response in our experiment may be related to the liberal infusion strategy with considerable volumes of fluid infused as prehydration prior to LPS 
administration and relatively high infusion rates during the endotoxemia experiment, thus preventing extensive fluctuations of perfusion pressure and cardiac output. This volume infusion is standard therapy in the local endotoxemia protocol to prevent vasovagal reactions [24]. Studies reporting enhanced dynamic cerebral autoregulation used a more conservative infusion regimen, with no additional fluid infusion prior to and during the entire experiment, versus approximately $2.5 \mathrm{~L}$ fluid infusion in our experiments [29].

Besides, it should be noted that in septic patients admitted to the intensive care, much higher plasma LPS concentrations (in the $\mathrm{mg} \mathrm{L}^{-1}$ range) are often observed [30-32].

In our sepsis patients, the VLF phase was significantly lower, but the gain was not different compared to healthy volunteers before and during endotoxemia. This is consistent with previous reports that the response of the cerebrovascular bed is slower in sepsis, but the magnitude of change is not affected [3,4,15,17,18,33,34]. A disturbed autoregulation could potentially, expose the brain to hypo- and hyperperfusion during acute alterations in ABP and contribute to the development of SAE. Perfusion as measured by the CBFV was highly variable in our sepsis patients and in line with the current literature that describes cerebral perfusion as increased, unchanged or decreased after sepsis [13-16,35] Comparison between these studies is hampered by the large variability in severity of the disease, timing of the measurements and techniques used to measure CBF.

The $\mathrm{CrCP}$ decreased during human endotoxemia and was low in sepsis, reflecting a decreased vascular tone in these patients. This indicates that a cerebrovascular adaptation process has been switched on, also during experimental endotoxemia with intact cerebral autoregulation. These results are analogous to our previous observations of a decreased $\mathrm{CrCP}$ in human endotoxemia (2 ng/kg bolus) and sepsis [20]. This decreased $\mathrm{CrCP}$ operates as a protection for the brain against ischemia, and is mainly caused by a decrease in the time constant (Tau) of the cerebral arterial bed (Tau $=C_{a} \times$ CVR). These data are in agreement with earlier studies where an increased pulsatility index was demonstrated in sepsis and associated with the development of SAE [35-37].

This study has some limitations. The first limitation pertains the small groups of subjects enrolled and the fact that sex and age where different between the endotoxemia and the sepsis groups. As described in a previous study, we enrolled only young healthy male people in the experimental human endotoxemia study to confine intersubject variability [20]. A second limitation is the measurement of MAP through a arterial catheter in the radial artery. To estimate perfusion of the brain, measurement of MAP in the MCA at brain level would be more accurate instead of in the radial artery at the heart level, but is not realizable. A third limitation is the estimation of $\mathrm{CrCP}$ by a mathematical model. This estimation has the risk of bias. Intracranial pressure is essential for the 
most reliable establishment of $\mathrm{CrCP}$, but clearly intracranial pressure was not measured in this study. Because intracranial pressure is low in sepsis, the absence of intracranial pressure data is assumed not to significantly influence the estimation of CrCP [38]. Fourth, cerebral perfusion changes may be more pronounced in some regions of the brain than in other regions of the brain. We measured CBFV MCA and derived $\mathrm{CrCP}$ from this CBFV MCA. So, using this method, a heterogeneous distribution cannot be established. Fifth, dynamic autoregulation was determined by TFA and performed according to the recommendations of the international CARNet to assess cerebral autoregulation in the frequency domain using artifact free segments of $5 \mathrm{~min}$. This is indeed in accordance wthe the CARNet white paper, however, it is arguable if such short segments can be used to assess VLF in septic patients, because the spectral power of ABP is incredibly low here. Sixth, we used two different batches of purified LPS in the two LPS groups. We can't exclude whether this has caused different LPS-induced immune/cytokine responses.

Finally, we cannot rule out the consequences of alterations in carbon dioxide on CBFV MCA and modification, because of an increase in respiratory rate and temperature after infusion of LPS [20].

\section{CONCLUSIONS}

Continuous LPS infusion ( $4 \mathrm{ng} / \mathrm{kg}$ over $3 \mathrm{~h}$ ) results in similar vasodilation and decrease in blood pressure, and also does not affect the cerebrovascular profile to a greater extent compared to bolus LPS administration ( $2 \mathrm{ng} / \mathrm{kg}$ ). Dynamic cerebral autoregulation remains intact during experimental human endotoxemia. In sepsis, a low phase with normal gain suggests a delayed cerebrovascular response to changes in blood pressure. $\mathrm{CrCP}$ decreases during endotoxemia and is low in sepsis patients, reflecting a decreased vascular tone. These results indicate an intact cerebral autoregulation in the human endotoxemia model with an active cardiovascular compensatory mechanism, whereas cerebral autoregulation is disturbed in sepsis patients, plausibly because the compensatory cardiovascular mechanism is exhausted. According to these data the human endotoxemia model cannot be used as a proxy for sepsis-related cerebrovascular research.

\section{ABBREVIATIONS}

$\begin{array}{ll}\text { ABP } & \text { arterial blood pressure } \\ \text { b-LPS } & \text { bolus LPS administration } \\ \text { BMI } & \text { body mass index } \\ C_{a} & \text { compliance of the vascular bed } \\ \text { CABV } & \text { cerebral arterial blood volume } \\ \text { CBF } & \text { cerebral blood flow } \\ \text { CBFV } & \text { cerebral blood flow velocity } \\ \text { BL } & \text { baseline } \\ \text { c-LPS } & \text { continuous LPS infusion }\end{array}$




$\begin{array}{ll}\text { CPP } & \text { cerebral perfusion pressure } \\ \text { CrCP } & \text { critical closing pressure } \\ \text { CVR } & \text { cerebrovascular resistance } \\ \text { HR } & \text { heart rate } \\ \text { IL-6 } & \text { interleukin } 6 \\ \text { IL-8 } & \text { interleukin } 8 \\ \text { IL-10 } & \text { interleukin 10 } \\ \text { IQR } & \text { interquartile range } \\ \text { LF } & \text { low frequency } \\ \text { LPS } & \text { lipopolysaccharide } \\ \text { MAP } & \text { mean arterial pressure } \\ \text { MFV } & \text { mean flow velocity } \\ \text { RASS } & \text { Richmond Agitation and Sedation Scale } \\ \text { SAE } & \text { sepsis-associated encephalopathy } \\ \text { SD } & \text { standard deviation } \\ \text { SEM } & \text { standard error mean } \\ \text { TCD } & \text { transcranial Doppler } \\ \text { TFA } & \text { transfer function analysis } \\ \text { TNFa } & \text { tumor necrosis factor a } \\ \text { VLF } & \text { very low frequency }\end{array}$

\section{DATA AVAILABILITY}

The dataset of the study is available from the authors upon reasonable request.

\section{AUTHOR CONTRIBUTIONS}

All authors designed the study. JB, GL, RS and $\mathrm{CH}$ performed the LPS experiments. JB and $\mathrm{CH}$ performed the sepsis experiments. JB, $\mathrm{CK}$ and $\mathrm{CH}$ analyzed the data. JB, $\mathrm{CK}$ and $\mathrm{CH}$ wrote the paper with input from all authors.

\section{CONFLICTS OF INTEREST}

The authors declare no conflicts of interest.

\section{REFERENCES}

1. Singer M, Deutschman CS, Seymour CW, Shankar-Hari M, Annane D, Bauer M, et al. The Third International Consensus Definitions for Sepsis and Septic Shock (Sepsis-3). JAMA. 2016;315(8):801-10.

2. Sonneville R, de Montmollin E, Poujade J, Garrouste-Orgeas M, Souweine B, Darmon $\mathrm{M}$, et al. Potentially modifiable factors contributing to sepsisassociated encephalopathy. Intensive Care Med. 2017;43(8):1075-84.

3. Berg RM, Plovsing RR, Ronit A, Bailey DM, Holstein-Rathlou NH, Moller K. Disassociation of static and dynamic cerebral autoregulatory performance in healthy volunteers after lipopolysaccharide infusion and in patients with sepsis. Am J Physiol Regul Integr Comp Physiol. 2012;303(11):R1127-35. 
4. Schramm P, Klein KU, Falkenberg L, Berres M, Closhen D, Werhahn KJ, et al. Impaired cerebrovascular autoregulation in patients with severe sepsis and sepsis-associated delirium. Crit Care. 2012;16(5):R181.

5. Crippa IA, Subira C, Vincent JL, Fernandez RF, Hernandez SC, Cavicchi FZ, et al. Impaired cerebral autoregulation is associated with brain dysfunction in patients with sepsis. Crit Care. 2018;22(1):327.

6. Claassen JA, Meel-van den Abeelen AS, Simpson DM, Panerai RB; International Cerebral Autoregulation Research Network. Transfer function analysis of dynamic cerebral autoregulation: A white paper from the International Cerebral Autoregulation Research Network. J Cereb Blood Flow Metab. 2016;36(4):665-80.

7. Dewey RC, Pieper HP, Hunt WE. Experimental cerebral hemodynamics. Vasomotor tone, critical closing pressure, and vascular bed resistance. J Neurosurg. 1974;41(5):597-606.

8. Suffredini AF, Fromm RE, Parker MM, Brenner M, Kovacs JA, Wesley RA, et al. The cardiovascular response of normal humans to the administration of endotoxin. N Eng J Med. 1989;321(5):280-7.

9. Berg RM, Plovsing RR, Evans KA, Christiansen CB, Bailey DM, Holstein-Rathlou $\mathrm{NH}$, et al. Lipopolysaccharide infusion enhances dynamic cerebral autoregulation without affecting cerebral oxygen vasoreactivity in healthy volunteers. Crit Care. 2013;17(5):R238.

10. Brassard P, Kim YS, van Lieshout J, Secher NH, Rosenmeier JB. Endotoxemia reduces cerebral perfusion but enhances dynamic cerebrovascular autoregulation at reduced arterial carbon dioxide tension. Crit Care Med. 2012;40(6):1873-8.

11. Berg RM, Plovsing RR, Bailey DM, Holstein-Rathlou NH, Moller K. Dynamic cerebral autoregulation to induced blood pressure changes in human experimental and clinical sepsis. Clin Physiol Funct Imaging. 2016;36(6):490-6.

12. Goodson CM, Rosenblatt K, Rivera-Lara L, Nyquist P, Hogue CW. Cerebral Blood Flow Autoregulation in Sepsis for the Intensivist: Why Its Monitoring May Be the Future of Individualized Care. J Intensive Care Med. 2018;33(2):63-73.

13. Bowton DL, Bertels NH, Prough DS, Stump DA. Cerebral blood flow is reduced in patients with sepsis syndrome. Crit Care Med. 1989;17(5):399-403.

14. Maekawa T, Fujii Y, Sadamitsu D, Yokota K, Soejima Y, Ishikawa T, et al. Cerebral circulation and metabolism in patients with septic encephalopathy. Am J Emerg Med. 1991;9(2):139-43.

15. Pfister D, Siegemund M, Dell-Kuster S, Smielewski P, Ruegg S, Strebel SP, et al. Cerebral perfusion in sepsis-associated delirium. Crit Care. 2008;12(3):R63.

16. Pierrakos C, Antoine A, Velissaris D, Michaux I, Bulpa P, Evrard P, et al. Transcranial doppler assessment of cerebral perfusion in critically ill septic patients: a pilot study. Ann Intensive Care. 2013;3:28.

17. Steiner LA, Pfister D, Strebel SP, Radolovich D, Smielewski P, Czosnyka M. Near-infrared spectroscopy can monitor dynamic cerebral autoregulation in adults. Neurocrit Care. 2009;10(1):122-8. 
18. Bindra J, Pham P, Chuan A, Jaeger M, Aneman A. Is impaired cerebrovascular autoregulation associated with outcome in patients admitted to the ICU with early septic shock? Crit Care Resusc. 2016;18(2):95-101.

19. Kiers D, Koch RM, Hamers L, Gerretsen J, Thijs EJ, van Ede L, et al. Characterization of a model of systemic inflammation in humans in vivo elicited by continuous infusion of endotoxin. Sci Rep. 2017;7:40149.

20. van den Brule JMD, Stolk R, Vinke EJ, van Loon LM, Pickkers P, van der Hoeven JG, et al. Vasopressors Do Not Influence Cerebral Critical Closing Pressure During Systemic Inflammation Evoked by Experimental Endotoxemia and Sepsis in Humans. Shock. 2018;49(5):529-35.

21. Levy MM, Fink MP, Marshall JC, Abraham E, Angus D, Cook D, et al. 2001 SCCM/ESICM/ACCP/ATS/SIS International Sepsis Definitions Conference. Intensive Care Med. 2003;29(4):530-8.

22. Dellinger RP, Levy MM, Carlet JM, Bion J, Parker MM, Jaeschke R, et al. Surviving Sepsis Campaign: international guidelines for management of severe sepsis and septic shock: 2008. Intensive Care Med. 2008;34(1):17-60.

23. Dorresteijn MJ, van Eijk LT, Netea MG, Smits P, van der Hoeven JG, Pickkers P. Iso-osmolar prehydration shifts the cytokine response towards a more anti-inflammatory balance in human endotoxemia. J Endotoxin Res. 2005;11(5):287-93.

24. Kiers D, van der Heijden WA, van Ede L, Gerretsen J, de Mast Q, van der Ven AJ, et al. A randomised trial on the effect of anti-platelet therapy on the systemic inflammatory response in human endotoxaemia. Thromb Haemost. 2017;117(9):1798-807.

25. Varsos GV, Kasprowicz M, Smielewski P, Czosnyka M. Model-based indices describing cerebrovascular dynamics. Neurocrit Care. 2014;20(1):142-57.

26. Varsos GV, Richards H, Kasprowicz M, Budohoski KP, Brady KM, Reinhard M, et al. Critical closing pressure determined with a model of cerebrovascular impedance. J Cereb Blood Flow Metab. 2013;33(2):235-43.

27. Moller K, Strauss GI, Qvist J, Fonsmark L, Knudsen GM, Larsen FS, et al. Cerebral blood flow and oxidative metabolism during human endotoxemia. J Cereb Blood Flow Metab. 2002;22(10):1262-70.

28. Andreasen AS, Krabbe KS, Krogh-Madsen R, Taudorf S, Pedersen BK, Moller K. Human endotoxemia as a model of systemic inflammation. Current Med Chem. 2008;15(17):1697-705.

29. Berg RM (Department of Clinical Physiology and Nuclear Medicine, Frederiksberg Hospital, Frederiksberg, Denmark). Personal Communication. 2018 Jun 7.

30. Behre G, Schedel I, Nentwig B, Wormann B, Essink M, Hiddemann W. Endotoxin concentration in neutropenic patients with suspected gramnegative sepsis: correlation with clinical outcome and determination of antiendotoxin core antibodies during therapy with polyclonal immunoglobulin M-enriched immunoglobulins. Antimicrob Agents Chemother. 1992;36(10):2139-46.

31. Opal SM, Scannon PJ, Vincent JL, White M, Carroll SF, Palardy JE, et al. Relationship between plasma levels of lipopolysaccharide (LPS) and LPS- 
binding protein in patients with severe sepsis and septic shock. J Infect Dis. 1999;180(5):1584-9.

32. Marshall JC, Walker PM, Foster DM, Harris D, Ribeiro M, Paice J, et al. Measurement of endotoxin activity in critically ill patients using whole blood neutrophil dependent chemiluminescence. Crit Care. 2002;6(4):342-8.

33. Terborg C, Schummer W, Albrecht M, Reinhart K, Weiller C, Rother J. Dysfunction of vasomotor reactivity in severe sepsis and septic shock. Intensive Care Med. 2001;27(7):1231-4.

34. Taccone FS, Castanares-Zapatero D, Peres-Bota D, Vincent JL, Berre J, Melot C. Cerebral autoregulation is influenced by carbon dioxide levels in patients with septic shock. Neurocrit Care. 2010;12(1):35-42.

35. Pierrakos C, Attou R, Decorte L, Kolyviras A, Malinverni S, Gottignies P, et al. Transcranial Doppler to assess sepsis-associated encephalopathy in critically ill patients. BMC Anesthesiol. 2014;14:45.

36. Fulesdi B, Szatmari S, Antek C, Fulep Z, Sarkany P, Csiba L, et al. Cerebral vasoreactivity to acetazolamide is not impaired in patients with severe sepsis. J Crit Care. 2012;27(4):337-43.

37. Szatmari S, Vegh T, Csomos A, Hallay J, Takacs I, Molnar C, et al. Impaired cerebrovascular reactivity in sepsis-associated encephalopathy studied by acetazolamide test. Crit Care. 2010;14(2):R50.

38. Pfister D, Schmidt B, Smielewski P, Siegemund M, Strebel SP, Ruegg S, et al. Intracranial pressure in patients with sepsis. Acta Neurochir Suppl. 2008;102:71-5.

How to cite this article:

van den Brule JMD, van Kaam CR, Leijte GP, Stolk R, Pickkers P, van der Hoeven JG, Kox M, Hoedemaekers CWE. Dynamic Cerebral Autoregulation and Critical Closing Pressure in Experimental Human Endotoxemia and Sepsis Patients. Med One. 2019;4:e190009. https://doi.org/10.20900/mo.20190009 